\title{
Diseño y validación de una herramienta para medir la percepción de las condiciones de trabajo: caso Sector manufacturero de la región Caribe colombiana*
}

\author{
Design and Validation of an Instrument to Measure the \\ Perception of Working Conditions: Case Manufacturing \\ Sector Colombian Caribbean Region
}

Recibido: 25 de junio de 2014 | Aceptado: 02 de febrero de 2016

\author{
LAURA Martínez Buelvas ** \\ OSCAR OvIEDO-TRESPALACIOS ${ }^{* * *}$ \\ CARMENZA LUNA AMAYA **** \\ Universidad del Norte, Barranquilla, Colombia
}

doi: 10.11144/Javeriana.upsy15-1.dvhm

Para citar este artículo: Martínez Buelvas, L., Oviedo-Trespalacios, O., \& Luna Amaya, C. (2016). Diseño y validación de una herramienta para medir la percepción de las condiciones de trabajo: caso Sector manufacturero de la región Caribe colombiana. Universitas Psychologica, 15(1), 339-348. http:// dx.doi.org/10.11144/Javeriana.upsy15-1.dvhm

* Artículo de investigación. COLCIENCIAS

** Profesor asociado Departamento de Ingeniería Industrial, Universidad del Norte, Colombia. Correo electrónico: buelvasl@uninorte.edu.co

**** PhD (c) Centre for Accident Research and Road Safety - Queensland (CARRS-Q), Institute of Health and Biomedical Innovation (IHBI), Queensland University of Technology (QUT), Australia Profesor Departamento de Ingeniería Industrial, Universidad del Norte, Colombia. Correo electrónico: oscar.oviedotrespalacios@qut.edu.au.

${ }^{* * * * *} \mathrm{PhD}$ profesor Departamento de Ingeniería Industrial, Universidad del Norte, Colombia. Correo electrónico: cluna@uninorte.edu.co

\section{RES U MEN}

El Cuestionario de Condiciones de Trabajo fue diseñado y aplicado como una herramienta de evaluación de la percepción de la seguridad en el trabajo en una muestra de 518 empleados de diferentes empresas del sector manufacturero de la región Caribe colombiana. Se realizaron análisis de confiabilidad, análisis factorial exploratorio y análisis de componentes principales con rotación VARIMAX. Los resultados exhiben una aceptable confiabilidad interna de la escala $(\alpha=0.793)$ y una adecuación satisfactoria de los datos a la matriz factorial $(\mathrm{KMO}=0.785)$. La estructura factorial quedó representada por 17 ítems escalonados en cinco grupos: carga física, ambiente térmico, ruido, riesgos laborales y seguridad en el trabajo. Finalmente, el instrumento confirma una confiabilidad adecuada de gran utilidad para la comprensión e implementación de estrategias de acción en cuanto a la realización de modificaciones en las condiciones de trabajo en el sector para lograr satisfacción entre los empleados.

Palabras clave

escala de condiciones de trabajo; seguridad en el trabajo; prácticas administrativas de seguridad en el trabajo; clima de seguridad; satisfacción en el trabajo

\section{A B S T R A C T}

The Working Conditions Survey was designed and applied as a tool for assessing psychosocial components of job safety in a sample of 518 employees from different companies in the manufacturing sector of the Colombian Caribbean region. Reliability analysis, exploratory factor analysis and principal components analysis with VARIMAX rotation were performed. Results show acceptable internal reliability of the scale $(\alpha=0.793)$ and satisfactory adequacy of factorial matrix data $(\mathrm{KMO}=0.785)$. The factorial structure was represented by 17 items staggered into five groups: physical load, thermal environment, noise, occupational hazards and safety at work. In conclusion confirms the instrument adequate reliability and structural stability useful for the analysis and design of programs in order to implement changes in working conditions to achieve employee satisfaction.

Keywords

work conditions scale; job safety; management safety practices; safety climate; work satisfaction 


\section{Introducción}

El trabajo se define como el medio a través del cual los seres humanos se relacionan e interactúan con el ambiente para conseguir los bienes y servicios necesarios para vivir (Acevedo, Farias, Sánchez, Astegiano, \& Fernández, 2012). Agregando a lo anterior, se ha dicho que éste satisface las necesidades propias y las de la comunidad, da independencia, permite organizar el tiempo, desarrollar una actividad creativa, adquirir una identidad social, garantizar una seguridad personal o familiar y es, en general, el medio para la realización personal (Acevedo et al., 2012). Ahora bien, conceptualizar a la calidad de vida laboral en los últimos años ha recuperado protagonismo a nivel mundial, surgiendo así diferentes enfoques, los cuales agregan a la tradicional preocupación por la productividad y competitividad económica un énfasis en la necesidad de inclusión social y mecanismos de resguardo de las condiciones de los empleados al interior de la organización.

La calidad de vida laboral (CVL) en los últimos años se ha convertido en los países desarrollados en el elemento de referencia denominado "estado de bienestar", concepto afectado de manera compleja por múltiples factores, entre ellos, el estado psicológico, el nivel de dependencia, las relaciones sociales, las características del ambiente y la salud física (Márquez Membrive et al., 2011). Adicionalmente se puede definir como la exigencia y el anhelo del empleado con lo que respecta a las condiciones de trabajo, la remuneración, las posibilidades de desarrollo profesional, el equilibrio entre el rol laboral y familiar, la seguridad y las interacciones sociales en el lugar de trabajo (Charu, 2012; Narehan, Hairunnisa, Norfadzillah, \& Freziamella, 2014; Selahattin \& Omer, 2012).

El término calidad de vida laboral (CVL) abarca una variedad de programas, técnicas, teorías y estilos de gestión, a través de los cuales se diseñan las organizaciones y los puestos de trabajos, a fin de conceder a los trabajadores mayor autonomía, responsabilidad y autoridad (Das \& Vijayalakshmi, 2013). Desde este punto de vista, la CVL es un concepto amplio y heterogéneo, dadas la riqueza y la pluralidad de los diferentes temas estrecha- mente relacionados con el mundo laboral, como consecuencia de las diversas disciplinas, enfoques teóricos y áreas de estudio desde el cual se puede abordar (Jagannathan \& Akhila, 2009; Segurado Torres \& Agulló Tomás, 2002).

Desde esta perspectiva, las condiciones de trabajo son consideradas como un factor determinante en los procesos de salud-enfermedad a los cuales se exponen los trabajadores (Martínez Buelvas, Oviedo Trespalacios, \& Luna Amaya, 2013). En un sentido amplio, las condiciones de trabajo se definen como cualquier aspecto circunstancial en el que se produce la actividad laboral, incluyendo tanto factores del entorno físico en el que se realiza como las circunstancias temporales en que se da (Acevedo et al., 2012; Akranavičiūtė \& Ruževičius, 2007; Pailhé, 2005). Se hace hincapié en la necesidad de mejorar las condiciones de trabajo para disminuir los riesgos asociados a la actividad laboral y con ello, mejorar no solo la seguridad y salud de los trabajadores, sino también su calidad de vida. Por otra parte, se considera que aparte de la retribución, el ambiente físico de trabajo y la seguridad laboral son factores de higiene, los cuales hacen referencia al contexto en las cuales se desarrolla el trabajo y las condiciones que lo rodean (Martínez Gamarra \& Ros Mar, 2010). En este contexto, las condiciones de trabajo son concebidas como el conjunto de circunstancias y características materiales, ecológicas, económicas, políticas, organizacionales, entre otras, a través de las cuales se efectúan las relaciones laborales. Estudios ponen de manifiesto que la configuración de este escenario repercute no sólo en la calidad de la vida laboral, sino también en el bienestar, salud, seguridad y motivación del empleado (Blanch, Sahagún, \& Cervantes, 2010; Llorente, Arechavala, \& Negro Macho, 2012).

En el actual escenario mundial de crisis económica, en donde las políticas gubernamentales se centralizan en lograr mayores tasas de empleo como mecanismo para iniciar el crecimiento económico y garantizar el bienestar social, el estudio de las condiciones de trabajo cobra un especial interés para los investigadores académicos, empresarios y, en especial, para el trabajador (Llorente et al., 2012). En Colombia, la persistencia de la tasa de 
desempleo superior al 10\% desde 2009, la existencia del trabajo informal, la discriminación laboral, el incumplimiento de los estándares laborales establecidos por el Gobierno, entre otros factores, son muestra de la complejidad de los problemas asociados al mundo del trabajo y la insuficiencia de los esfuerzos implementados por el Estado para superarlos (Chaparro Hernández \& Bernal Uribe, 2007). Adicionalmente, en la última década las actividades manufactureras en Colombia contribuyeron con un $14.5 \%$ en promedio al PIB nacional, evidenciando un mayor grado de desarrollo con respecto a otros sectores (Rebolledo, Duque, López, \& Velasco, 2013) asimismo, en el periodo de 2002-2010 se estimó que el sector proporcionó el $12.8 \%$ del empleo nacional, del cual el $90 \%$ se genera en las cabeceras municipales y un $10 \%$ en las zonas rurales (Rebolledo et al., 2013). No obstante, reportes del Ministerio del Trabajo ponen de manifiesto que el sector manufacturero es el segundo sector con mayores tasas de accidentalidad con una totalidad de 25.810 casos reportados para marzo de 2013 (Luna Sanchéz, 2012), siendo éste superado por el sector inmobiliario. Esto no solo tiene consecuencias para la salud y calidad de vida de los colombianos, sino que afecta la productividad empresarial (Oviedo-Trespalacios, Manjarres, $\&$ Peñabaena Niebles, 2013). Por tal motivo, en la medida en que se siga perpetuando en el país en materia laboral un panorama desolador, éste continuará siendo un motivo de frustración y no una fuente de realización.

La presente investigación pretende exponer la estructura y las propiedades psicométricas del Cuestionario de Condiciones de Trabajo, diseñado y validado especialmente como herramienta para medir la percepción de los componentes de las condiciones de trabajo que impactan en el talento humano del sector manufacturero en la región $\mathrm{Ca}$ ribe colombiana. Su contenido incluye aspectos de tales condiciones que, en función de su presencia e intensidad, pueden funcionar como factores protectores y promotores de salud, bienestar y calidad de vida laborales o bien, por el contrario, como factores de riesgo psicosocial. La mayoría de estudios sobre condiciones de trabajo en Colombia se limitan a la caracterización de los factores de riesgo y posibles correlaciones de estos con la gestión organizacional a manera de diagnóstico, dejando de lado la proyección de los hallazgos identificados (Chaparro Hernández \& Bernal Uribe, 2007; Tuesca Molina, 2005), por tal, si se evalúa el impacto de las condiciones de trabajo en la satisfacción, la salud y el bienestar de los empleados, es decir, en la CVL, anteponiendo los intereses individuales de la organización, se lograría minimizar la fragmentación y el sesgo en las evaluaciones.

\section{Método}

\section{Participantes}

Para este estudio se obtuvo información de 518 empleados de diferentes empresas del sector manufacturero de la región Caribe colombiana con edades comprendidas entre 17 y 68 años (media $=$ 37 y D.E. $=11.73$ ). Su selección se hizo de forma aleatoria en cada uno de los diversos subsectores y tamaños de empresas (grandes, medianas y Pymes), no obstante, se contó con el debido consentimiento

TABlA 1.

Relación sexo versus estrato socioeconómico de los encuestados

\begin{tabular}{lccc}
\hline \multirow{3}{*}{ SEXO } & Bajo & ESTRATO & Alto \\
\multirow{2}{*}{ Femenino } & 70 & Medio & 12 \\
& $41.92 \%$ & 85 & $7.19 \%$ \\
\multirow{2}{*}{ Masculino } & 189 & $50.90 \%$ & 28 \\
& $53.85 \%$ & 134 & $7.98 \%$ \\
\hline
\end{tabular}

Fuente: elaboración propia 
de los involucrados para la realización de la entrevista. Los encuestados pertenecen a diferentes niveles socioeconómicos, predominando el estrato medio en el sexo femenino y el estrato bajo en sexo masculino, como se puede apreciar en la Tabla 1.

\section{Variables de estudio}

Las condiciones de trabajo se definen como el conjunto de características del trabajo relacionadas con la seguridad, salud y calidad de vida en el empleo, las cuales le brindan al trabajador confianza para desempeñarse efectivamente dentro de la organización (Casas, Repullo, Lorenzo, \& Cañas, 2002; García-Herrero, Mariscal, García-Rodríguez, \& Ritzel, 2012; Poulston, 2009). Se tienen en cuenta las condiciones físicas, los factores de riesgo y los procedimientos de promoción de la salud y prevención de enfermedades o accidentes (Lundh, Lützhöft, Rydstedt, \& Dahlman, 2011).

En general, el estudio de las condiciones de trabajo representa una característica prominente del entorno laboral en donde se involucran temas económicos, sociales, políticos, tecnológicos, ergonómicos, entre otros, derivando gran importancia por el notable consenso en torno a unas dimensiones básicas relativas del ambiente físico y social, a la organización, características de las tareas, a la salud, la seguridad, entre otras (Blanch, et al., 2010).

Los factores estudiados para medir el impacto de las condiciones de trabajo en el sector manufacturero en la región Caribe colombiana fueron extraídos de una revisión documental y de herramientas previamente diseñadas a las cuales se les realizaron adaptaciones conforme a las necesidades de investigación (Casas et al., 2002; Hayes, Perander, Smecko, \& Trask, 1998; Stevenson et al., 2009).

Para el caso en el cual se evaluaban las condiciones de seguridad en el trabajo, se adaptó el instrumento desarrollado por Hayes et al (1998), a partir del cual se valoraban integralmente las dimensiones de la percepción de seguridad en el trabajo, dichas dimensiones se resumen en: a) la percepción global de la seguridad en el trabajo, b) la seguridad de los compañeros de trabajo, c) la seguridad del jefe, d) las prácticas de gestión en temas de seguridad y e) la satisfacción con el programa de seguridad establecido. No obstante, conforme a la clasificación emitida por Casas et al. (2002), se incluyeron las preguntas asociadas al ambiente físico al cual se encuentran expuestos los trabajadores con el fin de determinar los potenciales riesgos presentes en su labor diaria.

\section{Procedimiento de diseño del instrumento}

El cuestionario propuesto estuvo constituido por 68 ítems distribuidos de la siguiente manera: 14 preguntas sobre condiciones sociodemográficas, 44 sobre las percepciones de condiciones de trabajo y 10 preguntas de comportamiento. Las preguntas asociadas a las percepciones de condiciones de trabajo fueron contestado mediante una escala tipo Likert con valores comprendidos entre 1 y 10 , siendo 1 totalmente en desacuerdo y 10 totalmente de acuerdo.

El proceso de construcción del instrumento se describe conforme a las siguientes fases:

Fase 1: se optó por un tipo de estudio instrumental debido a que en este caso se analizaron las propiedades psicométricas de diferentes instrumentos de evaluación previamente definidos y se llevaron a cabo adaptaciones de los mismos (Alfaro Inzunza et al., 2014), estableciendo así las dimensiones de evaluación basadas en una revisión actualizada de la literatura (Hernandez Sampieri, Fernández Collado, \& Baptista Lucio, 1997; Zorrilla \& Torres, 1992).

La segunda etapa de la investigación, de acuerdo a los resultados obtenidos, fue del tipo multivariado, correlacional, transversal y no experimental, dado que se evidenció una fiel representación del fenómeno de estudio, en un momento dado de tiempo y sin manipulación de las variables (Bello-Villanueva et al., 2014; Bello-Villanueva, Palacio, Rodríguez-Díaz, \& Oviedo-Trespalacios, 2013; Hernandez Sampieri et al., 1997; Zorrilla \& Torres, 1992).

Fase 2: se realizó la construcción del instrumento cuya base conceptual provino de investigaciones anteriores con el fin de conocer los factores de condiciones de trabajo que desde la perspectiva del 
empleado podrían impactar en su calidad de vida y de esta manera analizar dicho impacto en el sector al cual se evaluó. La validez del constructo se realizó a través de acuerdo interjueces, quienes eligieron aquellos ítems que tuvieran validez de contenido muestral. Se consultó a cinco profesionales con experticia en la temática y en el diseño de pruebas psicométricas, pero solo se obtuvo respuesta de tres jueces provenientes de la Universidad del Norte, del Grupo de Investigaciones en Desarrollo $\mathrm{Hu}$ mano (GIDHUM), Departamento de Psicología y Grupo de Investigaciones en Productividad y Competitividad, Departamento de Ingeniera Industrial. Al final se escogieron solo los ítems indicados por este grupo de expertos.

Fase 3: en esta etapa se procedió con la aplicación del instrumento con un total de 68 ítems siguiendo las recomendaciones de las evaluaciones de expertos. La información fue recolectada y posteriormente analizada.

\section{Análisis de datos}

El análisis de la consistencia interna se obtuvo calculando el coeficiente de alfa de Cronbach y la evaluación de la validez de constructo se ejecutó por medio del análisis factorial exploratorio: análisis de componentes principales con rotación VARIMAX siguiendo la metodología descrita por Sierra, Oviedo-Trespalacios, Candelo y Soto (2015). Adicionalmente, se realizó un análisis de fiabilidad de cada componente. La valoración de las escalas se realizó mediante el análisis de los datos a través del Paquete Estadístico para Ciencias Sociales (SPSS), versión 19 para Windows.

\section{Resultados}

\section{Análisis de fiabilidad}

Mediante el coeficiente alfa de Cronbach se estimó la confiabilidad para el total de la escala de condiciones de trabajo, resultando un $\alpha=0.88$. Posteriormente se descartaron aquellos ítems que no aportaban a la confiabilidad de la escala, descartando por dimensión 27. Una vez realizado este procedimiento, se estimó nuevamente la confiabi- lidad, arrojando un alfa de Cronbach de $\alpha=0.80$ para la escala total de estudio.

En relación a los ítems de condiciones de trabajo que impactan en el talento humano en el sector manufacturero de la región Caribe colombiana, se agruparon en cinco subdimensiones: carga física, ambiente térmico, ruido, riesgos laborales y seguridad en el trabajo. La media obtenida en dicha escala fue de 5.83 (D.E. = 3.22) y la evaluación de homogeneidad fue elevada en todos los casos obteniéndose correlaciones de ítems total superiores a 0.60. La estimación de la consistencia interna para cada una de las subdimensiones fue de 0.820 para el caso de carga física, 0.793 para el ambiente térmico reportado en el trabajo, 0.81 para el ruido presente, 0.88 y 0.88 para los riesgos y seguridad en el trabajo respectivamente; la homogeneidad de la prueba fue adecuada con correlaciones mínimas superiores a 0.40. En la Tabla 2 se detallan media, desviación estándar, alfa de Cronbach, correlación mínima y correlación ítem total para cada uno de los ítems de la escala.

\section{Análisis estructura factorial}

La evaluación de la validez de constructo se realizó por medio del análisis factorial exploratorio: análisis de componentes principales con rotación VARIMAX. El empleo de este método de rotación estuvo motivado porque no se partía de una concepción a priori de la estructura factorial de la escala, por lo que no se presupuso que pudieran existir varios factores correlacionados, y en que se pretendía que la solución obtenida fuera lo más simple y estable posible (Alandete, Martínez, \& Nohales, 2013). La adecuación de los datos a la matriz factorial fue satisfactoria, se encontró un $\mathrm{KMO}=0,79 \mathrm{y}$ la prueba de esfericidad de Bartlett fue altamente significativa $\left(\mathrm{Chi}^{2}=4438.7\right.$; g.l. $\left.=136 ; p<0.001\right)$. Se extrajeron inicialmente 11 factores de los cuales finalmente fueron interpretables sólo cinco, los cuales se explican en un $73.128 \%$ con respecto a la varianza total. La estructura factorial se representa por 17 ítems escalonados en cinco grupos: carga física (3 ítems), ambiente térmico (3 ítems), ruido ( 3 ítems), riesgos laborales ( 3 ítems) y seguridad en el trabajo (5 ítems). 
TABLA 2.

Media, desviación estándar, alfa Cronbach, correlaciones minimas y correlaciones item total - Escala Condiciones de Trabajo

\begin{tabular}{|c|c|c|c|c|c|}
\hline Ítems & Media & D.E. & $\alpha$ Cronbach & Correlaciones & rItem-Total \\
\hline \multicolumn{6}{|l|}{ Subdimensión: carga física } \\
\hline $\begin{array}{l}\text { Cuento con el tiempo adecuado para descansar cuando } \\
\text { he realizado un levantamiento de carga continuo }\end{array}$ & 4.67 & 3.79 & \multirow{3}{*}{0.82} & \multirow{3}{*}{0.562} & 0.64 \\
\hline $\begin{array}{l}\text { La organización me capacita en temas referentes al ade- } \\
\text { cuado levantamiento de carga para evitar lesiones }\end{array}$ & 4.97 & 3.87 & & & 0.68 \\
\hline $\begin{array}{l}\text { Se me supervisa el uso de equipos de protección personal } \\
\text { y el adecuado levantamiento de carga }\end{array}$ & 5.02 & 3.88 & & & 0.71 \\
\hline Subdimensión: ambiente térmico & & & \multirow{5}{*}{0.79} & \multirow{4}{*}{0.410} & \\
\hline La temperatura en el área de trabajo es agradable & 6.65 & 3.21 & & & 0.70 \\
\hline $\begin{array}{l}\text { Los equipos de ventilación y acondicionamiento del aire } \\
\text { en el puesto de trabajo son suficientes }\end{array}$ & 6.89 & 3.14 & & & 0.77 \\
\hline $\begin{array}{l}\text { La vestimenta que utilizo es adecuada con relación a la } \\
\text { temperatura existente en el puesto de trabajo }\end{array}$ & 8.19 & 2.37 & & & 0.49 \\
\hline \multicolumn{5}{|l|}{ Subdimensión: ruido } & \\
\hline $\begin{array}{l}\text { En mi puesto de trabajo existen fuentes de ruido, conti- } \\
\text { nuas o variables, que me obligan a levantar la voz duran- } \\
\text { te una conversación }\end{array}$ & 5.35 & 3.42 & \multirow{3}{*}{0.81} & \multirow{3}{*}{0.559} & 0.63 \\
\hline $\begin{array}{l}\text { El ruido generado en el área de trabajo me produce dolo- } \\
\text { res de cabeza, estrés u otras molestias }\end{array}$ & 4.06 & 3.16 & & & 0.67 \\
\hline $\begin{array}{l}\text { El ruido generado en el área de trabajo me obliga a man- } \\
\text { tener niveles de concentración por encima de lo que } \\
\text { normalmente requiere el puesto }\end{array}$ & 4.56 & 3.36 & & & 0.67 \\
\hline \multicolumn{5}{|l|}{ Subdimensión: riesgo laboral } & \\
\hline Mi trabajo es peligroso & 3.90 & 3.21 & \multirow{3}{*}{0.88} & \multirow{3}{*}{0.654} & 0.82 \\
\hline Mi trabajo es arriesgado & 3.84 & 3.23 & & & 0.81 \\
\hline $\begin{array}{l}\text { Mi trabajo es altamente peligroso para la salud } \\
\text { Subdimensión: seguridad en el trabajo }\end{array}$ & 3.42 & 3.05 & & & 0.69 \\
\hline $\begin{array}{l}\text { En la empresa existe el reglamento de higiene y seguridad } \\
\text { laboral y éste se aplica conforme a lo establecido por Ley }\end{array}$ & 7.45 & 3.16 & & & 0.73 \\
\hline $\begin{array}{l}\text { La empresa cuenta con programas de promoción y pre- } \\
\text { vención en temas referentes a seguridad e higiene laboral }\end{array}$ & 7.04 & 3.27 & & & 0.73 \\
\hline $\begin{array}{l}\text { Constantemente presto atención a las normas de seguri- } \\
\text { dad establecidas por la empresa }\end{array}$ & 7.99 & 2.67 & 0.88 & 0.497 & 0.75 \\
\hline $\begin{array}{l}\text { Mi jefe inmediato permanentemente me está educando } \\
\text { en la importancia de las normas de seguridad estableci- } \\
\text { das por la compañía }\end{array}$ & 7 & 3.23 & & & 0.66 \\
\hline $\begin{array}{l}\text { Presto atención a las normas de seguridad y las aplico } \\
\text { según lo establecido por la empresa }\end{array}$ & 8.11 & 2.69 & & & 0.69 \\
\hline
\end{tabular}

Fuente: elaboración propia

Los criterios del análisis factorial fueron: a) la pregunta debe tener una carga ponderal igual o superior a 0.40 ; b) la pregunta se incluye en un solo factor, aquél en el que presente un mayor carga ponderal; c) debe haber congruencia conceptual entre todas las preguntas que se incluyen en un factor, y d) un factor debe estar conformado por tres o más preguntas. En la Tabla 3 se presenta el detalle de la solución factorial obtenida. 
TABLA 3.

Análisis factorial exploratorio por componentes principales y rotación VARIMAX - Escala Condiciones de Trabajo

\section{Subdimensión: carga física}

$$
\text { Pregunta asociada }
$$

Cuento con el tiempo adecuado para descansar cuando he realizado un levantamiento de carga continuo

La organización me capacita en temas referentes al adecuado levantamiento de carga para evitar lesiones

Se me supervisa el uso de equipos de protección personal y el adecuado levantamiento de carga

Subdimensión: ambiente térmico

La temperatura en el área de trabajo es agradable

Los equipos de ventilación y acondicionamiento del aire en el puesto de trabajo son suficientes

La vestimenta que utilizo es adecuada con relación a la temperatura existente en el puesto de trabajo

\section{Subdimensión: ruido}

En mi puesto de trabajo existen fuentes de ruido, continuas o variables, que me obligan a levantar la voz durante una conversación

El ruido generado en el área de trabajo me produce dolores de cabeza, estrés u otras molestias

El ruido generado en el área de trabajo me obliga a mantener niveles de concentración por encima de lo que normalmente requiere el puesto

Subdimensión: riesgo laboral

Mi trabajo es peligroso

Mi trabajo es arriesgado

Mi trabajo es altamente peligroso para la salud

\section{Subdimensión: seguridad en el trabajo}

En la empresa existe el reglamento de higiene y seguridad laboral y éste se aplica conforme a lo establecido por Ley

La empresa cuenta con programas de promoción y prevención en temas referentes a seguridad e higiene laboral

Constantemente presto atención a las normas de seguridad establecidas por la empresa

Mi jefe inmediato permanentemente me está educando en la importancia de las normas de seguridad establecidas por la compañía

Presto atención a las normas de seguridad y las aplico según lo establecido por la empresa

Fuente: elaboración propia

\section{Discusión}

En la actualidad se ha demostrado en la literatura una estrecha relación entre la CVL y la satisfacción hacia el trabajo (Somarriba Arechavala, Merino Llorente, Ramos Truchero, \& Negro Macho, 2010). La salud de los trabajadores es uno de los principales determinantes de esta relación que no solo impacta el bienestar de los trabajadores, sino que trae profundas consecuencias para la productividad $\begin{array}{lllll}\text { F1 } & \text { F2 } & \text { F3 } & \text { F4 } & \text { F5 }\end{array}$

0.80

0.84

0.83 
sistencia interna y validez del constructo, teniendo en cuenta que las variables asociadas a esta dimensión son las de mayor predicción en las intenciones comportamentales.

El Cuestionario de Condiciones de Trabajo se construye a partir de instrumentos validados que estudian la percepción de factores de riesgo laboral (Chaparro Hernández \& Bernal Uribe, 2007; Tuesca Molina, 2005). Las escalas estudiadas para medir el impacto de las condiciones de trabajo en el sector manufacturero en la región Caribe colombiana fueron extraídas de una revisión documental y de herramientas previamente diseñadas a las cuales se les realizaron adaptaciones conforme a las necesidades de investigación. Para el caso en el cual se evaluaban las condiciones de seguridad en el trabajo, se adaptó el instrumento desarrollado por Hayes et al (1998), a partir del cual se valoraban integralmente las dimensiones de la percepción de seguridad en el trabajo. No obstante, conforme a la clasificación emitida por Casas et al. (2002), se incluyeron las preguntas asociadas al ambiente físico al cual se encuentran expuestos los trabajadores con el fin de determinar los potenciales riesgos presentes en su labor diaria.

En efecto, los análisis realizados demuestran que las propiedades psicométricas del instrumento son adecuados e implican una evaluación valida y confiable, con consistencia interna alta y destacan su utilidad en la evaluación de la percepción de las condiciones de trabajo; además, los resultados del análisis factorial realizado ponen de manifiesto que el cuestionario tiene una composición multifactorial que permite obtener medidas específicas para cada uno de los cinco factores identificados, a saber, carga física, ambiente térmico, ruido, riesgos laborales y seguridad en el trabajo. La investigación reportó que la escala de condiciones de trabajo presenta una media de 5.38 (D. E. = 3.22) y un alfa de Cronbach de 0.79 lo que indica que este instrumento tiene un alto grado de confiabilidad, validando su uso para la recolección de datos; la evaluación de homogeneidad fue elevada en todos los casos obteniéndose correlaciones de ítems total superiores a 0.60. Adicional a esto, la adecuación de los datos a la matriz factorial fue satisfactoria, encontrándose un $\mathrm{KMO}=0.79$.
Por otra parte, sólo fueron interpretables cinco factores, los cuales se explicaron en un $73.13 \%$ con respecto a la varianza total. En contraste con otros estudios, como por ejemplo Hayes (1998), en donde se realiza un diseño y validación de un instrumento para medir la percepción de las condiciones de seguridad en el trabajo, se concluye que la seguridad que demuestra el jefe y las prácticas de gestión en temas de seguridad son las variables que mejor predicen la satisfacción de los empleados al interior de la organización con un alfa de Cronbach de 0.88 no distante al obtenido en el presente estudio, siendo este último de 0.79 . Por otra parte, Blanch et al. (2010) diseñaron un Cuestionario de Condiciones de Trabajo con el fin de evalaur los componentes psicosociales del entorno laboral en organizaciones de servicio, encontrando valores de alfa de Croncbach que oscilaban entre 0.83 y 0.97 en las diferentes escalas de medición. Es decir, la correlación existente entre los valores obtenidos en el alfa de Cronbach indica que el instrumento propuesto posee una alta fiabilidad en términos de consistencia interna. Por otra parte, el procedimiento metodológico adoptado para su construcción avala su validez de contenido y de constructo.

El instrumento desarrollado es sencillo tanto en su aplicación como en la interpretación de los resultados, dado que se analizan y evalúan fenómenos polifacéticos y complejos, como las variables de condiciones de trabajo que impactan significativamente en la calidad de vida laboral de los empleados y en su productividad, en un ámbito de particular interés, como el sector manufacturero en la región Caribe colombiana. En términos generales, puede concluirse que el cuestionario de condiciones de trabajo representado en la presente investigación contribuye a generar una herramienta de gran utilidad psicométrica para la evaluación y diagnóstico de las variables que tienen mayor impacto en la calidad de vida laboral del talento humano, promoviendo a su vez la elección de estrategias de acción efectivas en cuanto a la realización de modificaciones en las condiciones de trabajo para lograr mejoras en la satisfacción de los empleados. Sin embargo, para investigaciones futuras se hace 
evidente la necesidad de nuevos estudios confirmatorios en cuanto a validez y fiabilidad en diferentes sectores productivos.

Se espera continuar con este proceso de validación en estudios comparativos utilizando otros cuestionarios psicométricos para así ajustar y reducir el número de ítems, minimizar el tiempo de administración de la escala y facilitar su aplicación.

\section{Limitaciones y futuras investigaciones}

Cabe resaltar la importancia del presente instrumento dado que ni en el país ni en el sector se han realizado investigaciones sobre esta temática en las cuales se crucen, por primera vez, variables como condiciones de trabajo y CVL, por lo cual no hay resultados con los cuales contrastar; sin embargo, éste es un paso para seguir trabajando en herramientas o instrumentos robustos que permitan aplicar métodos estadísticos complejos para el análisis de la información (Martínez, 2014).

\section{Referencias}

Acevedo, G., Farias, A., Sánchez, J., Astegiano, C., \& Fernández, A. (2012). Condiciones de Trabajo del Equipo de Salud en centros de Atencion Primaria desde la Perspectiva del Trabajo Decente. Revista Argentina Salud Pública, 3(12), 15-22.

Akranavičiūtè, D., \& Ruževičius, J. (2007). Quality of Life and its Components' Measurement. Gyvenimo kokybè ir jos sudedamuju daliu vertinimas, 52(2), 44-49.

Alandete, J. G., Martínez, E. R., \& Nohales, P. S. (2013). Estructura factorial y consistencia interna de una versión española del Purpose-In-Life Test. Universitas Psychologica, 12(2), 517-530.

Alfaro Inzunza, J., Guzmán Piña, J., García Gómez, C., Sirlopú Díaz, D., Oyanedel Sepúlveda, J. C., \& Gaudlitz Ruiz, L. (2015). Propiedades psicométricas de la escala Breve Multidimensional de Satisfacción con la Vida para Estudiantes (BMSLSS) en población infantil chilena (10-12 años). Universitas Psychologica, 14(1) 29-41.

Bello-Villanueva, A. M., Oviedo-Trespalacios, O., VeraVillarroel, P., Oviedo, O., Rodriguez-Diaz, M.,
Celis-Atenas, K., \& Pavez, P. (2014). Presentation of an attitudes and beliefs scale to assess adolescent sexuality and reproductive health of young males in the Caribbean region. Universitas Psychologica, 13(1), 47-60. http://dx.doi.org/10.11144/Javeriana. UPSY13-1.peea

Bello-Villanueva, A. M., Palacio, J., Rodríguez-Díaz, M., \& Oviedo-Trespalacios, O. (2013). Adolescent sexual behavioral intentions measurement: A gender approach in the Colombian Caribbean region. Terapia Psicologica, 31(3), 343-353.

Blanch, J. M., Sahagún, M., \& Cervantes, G. (2010). Estructura factorial del cuestionario de condiciones de trabajo. Revista de Psicología del Trabajo y de las Organizaciones, 26(3), 175-189.

Casas, J., Repullo, J. R., Lorenzo, S., \& Cañas, J. J. (2002). Dimensiones y medición de la calidad de vida laboral en profesionales sanitarios. Revista de administración sanitaria, 6(23), 143-160.

Chaparro Hernández, S. R., \& Bernal Uribe, C. (2007). Trabajo digno y decente en Colombia: Seguimiento y control preventivo a las políticas públicas. Bogotá: Procuraduría General de la Nación.

Charu, M. (2012). Occupational Stress and its impact on QWL with specific reference to Hotel Industry. Advances in Management, 5(9), 50-54.

Das, T. V., \& Vijayalakshmi, Ch. (2013). Quality of Work Life - A Strategy for Good Industrial Relations. Advances in Management, 6(11), 8-15.

García-Herrero, S., Mariscal, M. A., García-Rodríguez, J., \& Ritzel, D. O. (2012). Working conditions, psychological/physical symptoms and occupational accidents. Bayesian network models. Safety Science, 50(9), 1760-1774. http://dx.doi.org/10.1016/j. ssci.2012.04.005

Hayes, B. E., Perander, J., Smecko, T., \& Trask, J. (1998). Measuring Perceptions of Workplace Safety: Development and Validation of the Work Safety Scale. Journal of Safety Research, 29(3), 145-161.

Hernandez Sampieri, R., Fernández Collado, C., \& Baptista Lucio, P. (1997). Metodología de la Investigación. México: MCGraw-Hill Ed. Jagannathan, L., \& Akhila, P. R. (2009). Predictors of Quality of Work Life of Sales Force in Direct Selling Organizations. ICFAI Journal of Management Research, 8(6), 51-59. 
Llorente, M. C. M., Arechavala, N. S., \& Negro Macho, A. M. (2012). Un análisis dinámico de la calidad del trabajo en España. Los efectos de la crisis económica. Estudios De Economía Aplicada, $30(1), 261-282$.

Luna Sanchéz, D. (2012). Riesgos Laborales: Boletín No 5. Bogotá: Ministerio del Trabajo de Colombia.

Lundh, M., Lützhöft, M., Rydstedt, L., \& Dahlman, J. (2011). Working conditions in the engine department - A qualitative study among engine room personnel on board Swedish merchant ships. Applied Ergonomics, 42(2), 384-390. http://dx.doi. org/10.1016/j.apergo.2010.08.009

Márquez Membrive, J., Granero-Molina, J., Solvas Salmerón, M. J., Fernández-Sola, C., Rodríguez López, C. M., \& Parrón Carreño, T. (2011). Quality of life in perimenopausal women working in the health and educational system. Revista Latino-Americana de Enfermagem, 19(6), 1314-1321.

Martínez Buelvas, L., Oviedo Trespalacios, O., \& Luna Amaya, C. (2013). Condiciones de Trabajo que impactan en la Calidad de Vida Laboral. Salud Uninorte, 29(3) 542-560.

Martínez Gamarra, M., \& Ros Mar, R. (2010). De la calidad de vida laboral a los riesgos Psicosociales: Evaluación de la calidad de vida laboral. Salud Uninorte (28), 5-55.

Martínez, L. (2014). Evaluación Del Impacto De Las Condiciones De Trabajo En La Calidad De Vida Laboral En El Sector Manufacturero De La Región Caribe Colombiana (Trabajo de grado Maestría en Ingeniería Industrial), Universidad del Norte.

Narehan, H., Hairunnisa, M., Norfadzillah, R. A., \& Freziamella, L. (2014). The Effect of Quality of Work Life (QWL) Programs on Quality of Life (QOL) Among Employees at Multinational companies in Malaysia. Procedia - Social and Behavioral Sciences, 112, 24-34. http://dx.doi.org/10.1016/j. sbspro.2014.01.1136

Oviedo-Trespalacios, O., Manjarres, R., \& Peñabaena Niebles, R. (2013). The Learning Curve applied to fire extinguisher training: A comparison among different type of fires. Paper presented at the 22nd International Conference on Production Research, ICPR 2013.
Pailhé, A. (2005). Working Conditions: How Are Older Workers Protected in France? Population (English Edition, 2002-)(1/2), 93. http://dx.doi. org/10.2307/4148203

Poulston, J. M. (2009). Working Conditions in Hospitality: Employees' Views of the Dissatisfactory Hygiene Factors. Journal of Quality Assurance in Hospitality $\mathscr{E}$ Tourism, 10(1), 23-43. http://dx.doi. org/10.1080/15280080902716993

Rebolledo, J., Duque, C., López, L., \& Velasco, A. (2013). Perfil del sector manufacturero Colombiano. Magazín Empresarial, 9(19), 49-61.

Segurado Torres, A., \& Agulló Tomás, E. (2002). Calidad de vida laboral: hacia un enfoque integrador desde la psicología social. Psicothema, 14(4), 828 836. Selahattin, K., \& Omer, S. (2012). An Empirical Research on Relationship Quality of Work Life and Work Engagement. Procedia - Social and Behavioral Sciences, 62, 360-366. http://dx.doi. org/10.1016/j.sbspro.2012.09.057

Sierra, R. C., Oviedo-Trespalacios, O., Candelo, J. E., \& Soto, J. D. (2015). The influence of atmospheric conditions on the leakage current of ceramic insulators on the Colombian Caribbean coast. Environmental Science and Pollution Research, 22(4), 2526 2536. http://dx.doi.org/10.1007/s11356-014-3729-3

Somarriba Arechavala, N., Merino Llorente, M. C., Ramos Truchero, G., \& Negro Macho, A. (2010). La calidad del trabajo en la Union Europea. (The Quality of Job in the European Union. With English summary.). Estudios De Economía Aplicada, 28(3). Recuperado de http://www.revista-eea.net

Stevenson, R. J., Case, T. I., Porzig-Drummond, R., Oaten, M. J., Hodgson, D., \& Barouei, J. (2009). A scale for measuring hygiene behavior: Development, reliability and validity. American Journal of Infection Control, 37(7), 557-564. http://dx.doi. org/10.1016/j.ajic.2009.01.003

Tuesca Molina, R. (2005). La Calidad de vida, su importancia y cómo medirla: Universidad del Norte (Barranquilla, Colombia).

Zorrilla, S., \& Torres, M. (1992). Guía para elaborar la tesis. México: McGraw Hill. 